\title{
Genotypic, phenotypic, biochemical, physiological and pathogenicity-based categorisation of Acanthamoeba strains
}

\author{
Naveed Ahmed Khan ${ }^{1}$ and Noor Khan Tareen ${ }^{2}$ \\ ${ }^{1}$ School of Biological and Chemical Sciences, Birkbeck College, University of London, London, WC1E 7HX, England, UK; \\ ${ }^{2}$ District Headquarters Hospital, Bahawalnagar, Pakistan
}

Key words: Acanthamoeba, cytotoxicity, differentiation, keratitis, corneal epithelial cells

\begin{abstract}
The genus Acanthamoeba includes more than 20 morphological species, but classification is problematical. Recently, the discovery of substantial interstrain differences in ribosomal DNA (rDNA) sequences has prompted questions about the relatedness of strains of the same species. In this study, therefore, we have investigated relationships between two isolates of $A$. polyphaga, CCAP 1501/3c and ATCC 30871, using morphological, biochemical, physiological, molecular and cytotoxicity assays. We observed that $A$. polyphaga ATCC 30871 exhibited up to six arms in endocyst while A. polyphaga CCAP 1501/3c exhibited a maximum of 5 arms thus indicating their position in group 2 and 3, respectively. Acanthamoeba polyphaga ATCC 30871 exhibited growth at $37^{\circ} \mathrm{C}$ and growth on $1 \mathrm{M}$ mannitol plates while $A$. polyphaga CCAP $1501 / 3 \mathrm{c}$ did not. In addition, both isolates exhibited differences in isoenzyme banding patterns and rDNA restriction fragment polymorphisms. More importantly, A. polyphaga ATCC 30871 produced cytotoxicity on corneal epithelial cells while A. polyphaga CCAP 1501/3c had no effects, suggesting differences in pathogenicity. Thus, all the results provide evidence for significant differences between the strains and further provided the basis for reclassification of the isolates. Implications of these results in the clinical diagnosis of pathogenic Acanthamoeba are discussed.
\end{abstract}

Species of the genus Acanthamoeba Volkonsky, 1931 are present in diverse environments including swimming pools, surface waters, thermally polluted discharges, ocean waters, soil, air and tap waters. Based on epidemiological information to date, they can be considered as waterborne pathogens (De Jonckheere 1980, 1983, Marciano-Cabral 2000, Khan et al. 2001).

Although the species of Acanthamoeba can cause fatal infections involving brain (Gonzalez et al. 1986, Di Gregorio et al. 1991, Friedland et al. 1992, Gordon et al. 1992), however, they are most frequently associated with eye keratitis (Auran et al. 1987, John 1993, Badenoch et al. 1994, Niederkorn et al. 1999). Acanthamoeba keratitis is characterised by intense pain and a slowly worsening clinical course. If not diagnosed early and treated aggressively, the infection may spread to deep stroma and to other ocular tissues (Yang et al. 1997, Niederkorn et al. 1999). At present, diagnosis of the disease is not straightforward and treatment is problematic, consisting of hourly topical application of a combination of drugs for extended periods of time. In view of the devastating nature of the disease and the problems associated with the therapy, our goals are to find means to rapidly identify Acanthamoeba species and to differentiate pathogenic and non-pathogenic species/isolates. We have recently shown the use of morphological characters (Khan 2001), osmotolerance,
PCR (Khan et al. 2001), and extracellular proteases (Khan et al. 2000), to differentiate pathogenic and nonpathogenic Acanthamoeba strains. Our results directly correlated an isolate's pathogenic potential in its ability to produce cytotoxicity on corneal epithelial cells using in vitro assays, suggesting the potential use of these assays in clinical diagnosis of Acanthamoeba.

Attempts to correlate pathogenicity with species have proven difficult due to inconsistencies in the morphological-based classification. Recently, the genus Acanthamoeba has been reclassified into 13 different genotypes based on rDNA sequence analyses i.e., T1-T12 and T14 (Stothard et al. 1998, Gast 2001). However, two strains of $A$. polyphaga (Puschkarew) Page, 1967, A. polyphaga ATCC 30871 and A. polyphaga CCAP $1501 / 3 \mathrm{c}$, exhibited significant rDNA sequence differences between these two isolates and A. polyphaga ATCC 30871 was placed in T4 clade (mostly pathogens) and A. polyphaga CCAP $1501 / 3 \mathrm{c}$ was regrouped with A. palestinensis (Reich) Page, 1967 and placed in T2 clade (mostly non-pathogens). The aims of our study were to determine whether other morphological, physiological, biochemical, molecular and in vitro assays support the findings based on rDNA sequencing in order to differentiate these two isolates and whether these differences can be correlated with their pathogenic potential in order to develop methods for clinical diagnosis of pathogenic strains of Acanthamoeba. 


\section{MATERIALS AND METHODS}

Chemicals. All chemicals were purchased from Sigma Laboratories (St. Louis, MO) unless otherwise stated.

Culture of amoebae. Two strains of Acanthamoeba, A. polyphaga CCAP $1501 / 3 \mathrm{c}$ (isolated from old distilled water carboy, Culture Collection of Algae and Protozoa) and $A$. polyphaga ATCC 30871 (freshwater isolate, American Type Culture Collection) were used. Parasites were routinely grown in PYG medium [proteose peptone $0.75 \%(\mathrm{w} / \mathrm{v})$, yeast extract $0.75 \%(\mathrm{w} / \mathrm{v})$ and glucose $1.5 \%(\mathrm{w} / \mathrm{v})]$ at $30^{\circ} \mathrm{C}$.

Cytotoxicity assays using corneal epithelial cells. To determine the pathogenic potential of the Acanthamoeba isolates, cytotoxicity assays were performed as previously described (Khan et al. 2000). Briefly, immortalised rabbit corneal epithelial cells were grown to monolayers in 24-well plates. Both amoeba isolates $\left(10^{6}\right.$ per well) were incubated with corneal epithelial cells for up to $12 \mathrm{~h}$. Cytopathic effects were observed visually after haematoxylin staining. In addition, supernatants were collected and cytotoxicity determined by measuring lactate dehydrogenase (LDH) release (using Cytotoxicity detection kit, Roche, Indianapolis, IN). Percentage cytotoxicity was determined as follows: (sample value - control value / total LDH release - control value) $\times$ 100. Corneal epithelial cells incubated alone were used as controls. Total LDH release was determined from cells treated with $1 \%$ Triton $\mathrm{X}-100$ for $30 \mathrm{~min}$ at $37^{\circ} \mathrm{C}$. Each experiment was performed in triplicates and repeated at least three times.

Analyses of cyst morphology. Cyst morphology has been used in differentiation of Acanthamoeba species (Pussard and Pons 1977, Visvesvara 1991). In order to determine whether cyst morphology can be used to differentiate the amoeba isolates tested in this study, cyst morphology analyses assays were performed. Briefly, both Acanthamoeba isolates were inoculated on non-nutrient agar plates overlaid with Klebsiella aerogenes as previously described (Khan et al. 2001). Plates were observed daily for growth of amoeba trophozoites (vegetative infective form), followed by transformation into cysts (dormant form). Semi-thin sections of the cysts were made and stained using haematoxylin and observed microscopically.

Osmotolerance and temperature tolerance assays. Previously we have shown that osmolarity can be used as a marker to differentiate pathogenic and non-pathogenic Acanthamoeba strains (Khan et al. 2001). Osmolarity assays were performed to determine whether the amoeba isolates used in this study can be differentiated based on osmolarity. Briefly, amoeba isolates were inoculated on non-nutrient agar plates overlaid with $K$. aerogenes and containing $1 \mathrm{M}$ mannitol ( 0.25 osmolar in contrast to 0.025 osmolar without mannitol). Plates were incubated at $30^{\circ} \mathrm{C}$ for up to $72 \mathrm{~h}$. For temperature tolerance assays, amoebae were inoculated onto non-nutrient agar plates overlaid with $K$. aerogenes and incubated at $37^{\circ} \mathrm{C}$ for up to $72 \mathrm{~h}$. Growth of the organisms was observed microscopically as well as by measuring the diameter of clearance in the bacterial lawn (Khan et al. 2001).

Isoenzyme analyses. Both Acanthamoeba isolates $(5 \times$ $10^{7}$ ) were harvested by centrifugation at $800 \times g$ for $10 \mathrm{~min}$.
Pelleted cells were resuspended in cold phosphate buffered saline and centrifuged again at $800 \times g$ for $10 \mathrm{~min}$. This process was repeated twice. The washed cell pellet was then resuspended in an equal volume of enzyme stabiliser solution [Stock enzyme stabiliser, $1000 \times$ conc. $(200 \mathrm{mM}$ ethylene diamine tetra-acetic acid, $\mathrm{pH} 7.0$ and $200 \mathrm{mM}$ dithiothreitol and, once dissolved, $200 \mathrm{mM}$ of $\varepsilon$-amino-caproic acid was added)]. The suspension was freeze-thawed $3 \times$ in liquid $\mathrm{N}_{2}$ and centrifuged at $20,000 \times g$ for $20 \mathrm{~min}$ at $4^{\circ} \mathrm{C}$. The supernatant was electrophoresed on cellulose acetate gels $(76 \mathrm{~mm} \times$ $76 \mathrm{~mm} \operatorname{Titan}^{\circledR}$ III cellulose acetate plates, Helena Laboratories, England) according to manufacturer's instructions. Electrophoresis was performed at room temperature at 200 volts for $25 \mathrm{~min}$. Following electrophoresis, enzymatic activities of esterase (ES), malate dehydrogenase (MDH), phosphoenol pyruvate dehydrogenase (PEPD), isocitrate dehydrogenase (IDH), aldehyde oxidase (AO), xanthine dehydrogenase (XDH), glucosephosphoisomerase (GPI) and phosphoglucomutase (PGM) were determined. The reproducibility and position of the isoenzyme patterns were confirmed with different extracts.

Amplified ribosomal DNA restriction analyses (ARDRA). Restriction enzyme analyses were performed as described previously (Kong and Chung 1996). Briefly, PCR was performed using primers 5'-TTTGAATTCGCTCCA ATAGCGTATATTAA and 5'-TTTGAATTCAGAAAGAGC TATCAATCTGT in a volume of $50 \mu 1$ containing $1.25 \mathrm{U}$ Taq polymerase (Amersham, La Jolla, CA), 0.1-1.0 ng DNA (measured using Gene Quant, Amersham), $200 \mu \mathrm{M}$ dNTPs, 4 $\mathrm{mM} \mathrm{MgCl}_{2}$ and $0.5 \mu \mathrm{M}$ primer. The PCR reactions were performed at $94^{\circ} \mathrm{C}$ for $1 \mathrm{~min}, 55^{\circ} \mathrm{C}$ for $1 \mathrm{~min}, 72^{\circ} \mathrm{C}$ for $2 \mathrm{~min}$ for 30 cycles and final elongation step of $10 \mathrm{~min}$ at $72^{\circ} \mathrm{C}$. Amplified DNA was electrophoresed on a $2 \%$ agarose gel prepared in $1 \times$ TAE buffer $(0.04 \mathrm{M}$ Tris-acetate; $0.001 \mathrm{M}$ EDTA). Gels were stained using $0.5 \mu \mathrm{g} / \mathrm{ml}$ of ethidium bromide and visualised under UV illumination.

For restriction enzyme analyses, amplified DNA was extracted from agarose gels using a rapid gel extraction system (Invitrogen, Carlsbad, CA). All procedures were performed according to the manufacturer's instructions using AluI, CfoI, and Hinfl restriction enzymes.

\section{RESULTS}

\section{Acanthamoeba polyphaga strain ATCC 30871 but not CCAP 1501/3c produced corneal epithelial cell cytotoxicity}

To determine pathogenic potential of A. polyphaga ATCC 30871 and CCAP 1501/3c, cytotoxicity assays were performed using corneal epithelial cells. We determined that $A$. polyphaga ATCC 30871 produced cytopathic effects within $12 \mathrm{~h}$ as observed visually (Fig. 1A), as well as by measuring LDH release (Fig. 1B). In contrast, A. polyphaga CCAP $1501 / 3 \mathrm{c}$ did not exhibit cytotoxicity on corneal epithelial cells (Fig. 1A, B). Based on these data, A. polyphaga ATCC 30871 was considered as pathogenic while A. polyphaga CCAP $1501 / 3 \mathrm{c}$ as non-pathogenic. 
A

Cells alone A.polyphaga CCAP A.polyphaga ATCC

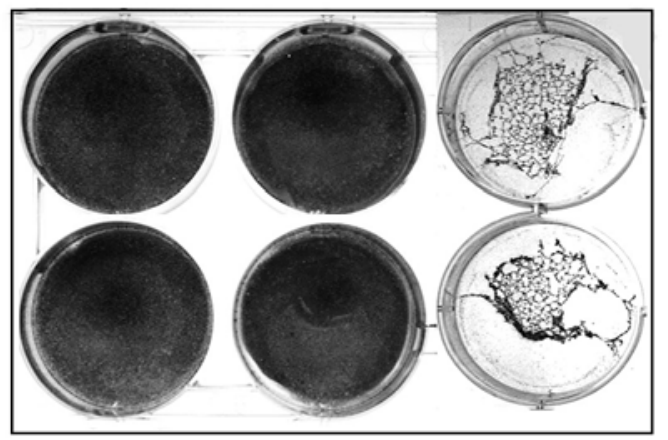

B

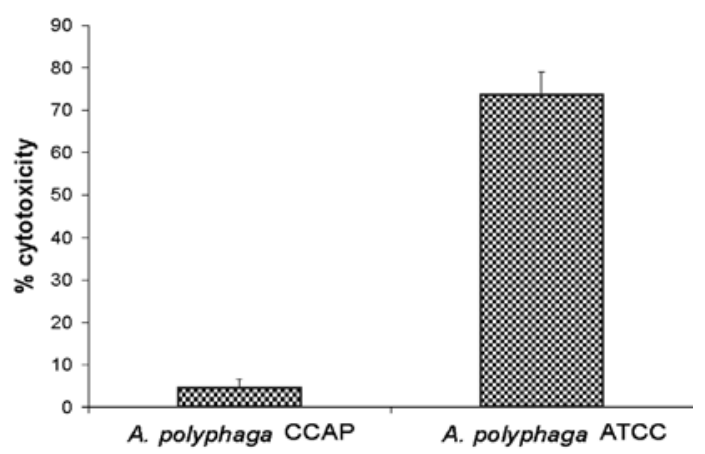

Fig. 1. Pathogenic Acanthamoeba strains produced cytotoxicity on corneal epithelial cells. A - Representative effects of $A$. polyphaga on corneal epithelial cells after $12 \mathrm{~h}$. Amoebae $\left(10^{6}\right)$ were incubated with epithelial cells in 24-well plates in serum free media as described in "Materials and Methods". Plates were incubated at $37^{\circ} \mathrm{C}$ in a $5 \% \mathrm{CO}_{2}$ incubator and cytotoxicity assessed by haematoxylin staining. Note that epithelial cells incubated with $A$. polyphaga ATCC 30871 exhibited disrupted monolayers while epithelial cells incubated with $A$. polyphaga CCAP $1501 / 3 \mathrm{c}$ remained intact. B - Following $12 \mathrm{~h}$ incubation as described above, supernatants were collected and LDH release was determined and converted to percentage cytotoxicity as described in "Materials and Methods". Note that $A$. polyphaga ATCC 30871 produced cytotoxicity on epithelial cells while A. polyphaga CCAP $1501 / 3 \mathrm{c}$ did not. Results represent mean of three independent experiments and each experiment was performed in triplicate. Error bars represent standard deviation.

Acanthamoeba polyphaga strains ATCC 30871 and CCAP $1501 / 3 c$ can be differentiated based on cyst morphology

Previously, Acanthamoeba isolates have been divided into three groups, based on cyst morphology (Pussard and Pons 1977, Visvesvara 1991). In this study, we determined whether $A$. polyphaga ATCC 30871 and $A$. polyphaga CCAP $1501 / 3$ c can be differentiated based on cyst morphology. We observed that cysts of A. polyphaga ATCC 30871 and CCAP 1501/3c
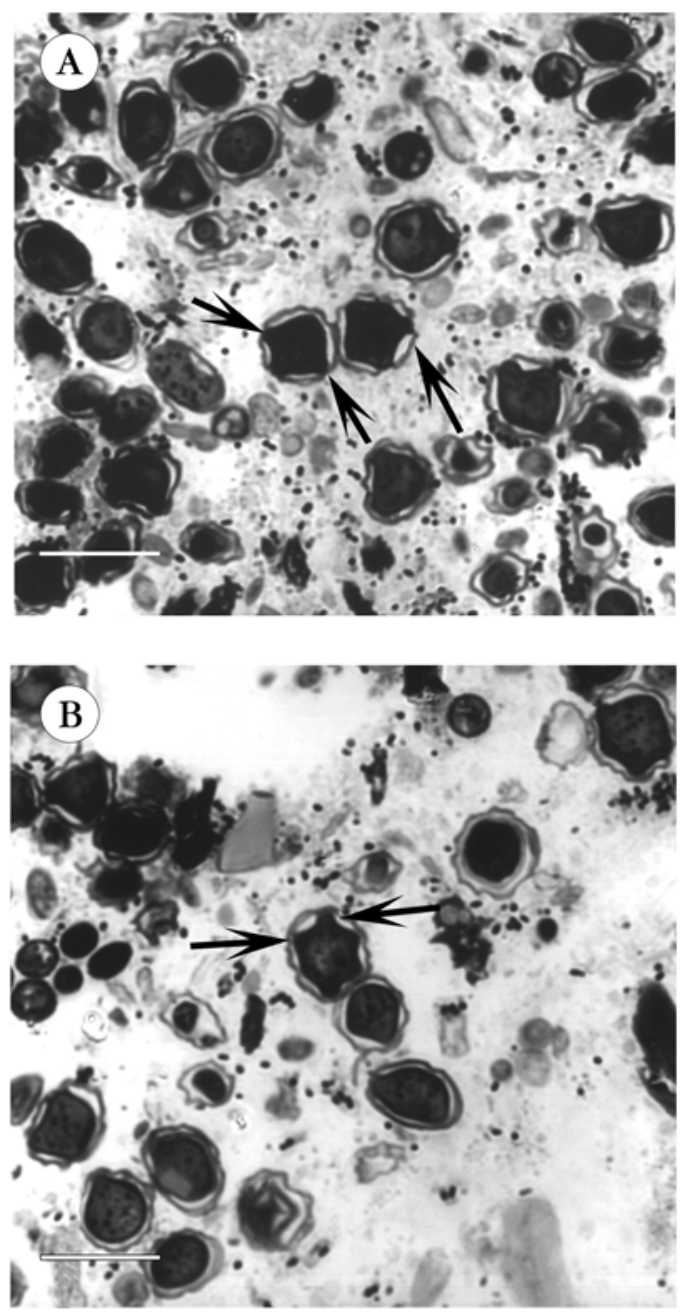

Fig. 2. Acanthamoeba polyphaga ATCC 30871 and CCAP $1501 / 3 \mathrm{c}$ are distinct based on cyst morphology. Cysts of $A$. polyphaga CCAP 1501/3c (A) or A polyphaga ATCC 30871 (B) were prepared and stained as described in "Materials and Methods". Note that $A$. polyphaga ATCC 30871 showed a maximum of 6 arms (group 2 isolates characteristic) while $A$. polyphaga CCAP 1501/3c exhibited up to 5 arms (group 3 isolates characteristic). Results are representative of three independent experiments. Scale bar $=15 \mu \mathrm{m}$.

are distinct, clearly indicating that both strains belong to distinct species (Fig. 2). Acanthamoeba polyphaga ATCC 30871 cysts exhibited a smooth outer cyst wall (ectocyst) that was separated from the inner cyst wall (endocyst). The endocyst was connected with the ectocyst at a certain number of extensions (known as arms or rays). The maximum number of arms determined in A. polyphaga CCAP $1501 / 3 \mathrm{c}$ was five (Fig. 2A), thus compatible with Group 3 isolates (Pussard and Pons 1977, Visvesvara 1991). In contrast the number of arms in $A$. polyphaga ATCC 30871 was six (Fig. 2B) indicating its position in Group 2 (Pussard and Pons 1977, Visvesvara 1991). All results are representative of three independent experiments. 


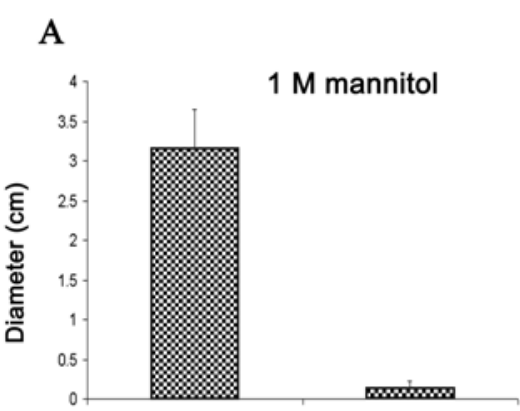

A. polyphaga ATCC A. polyphaga CCAP

\section{B}
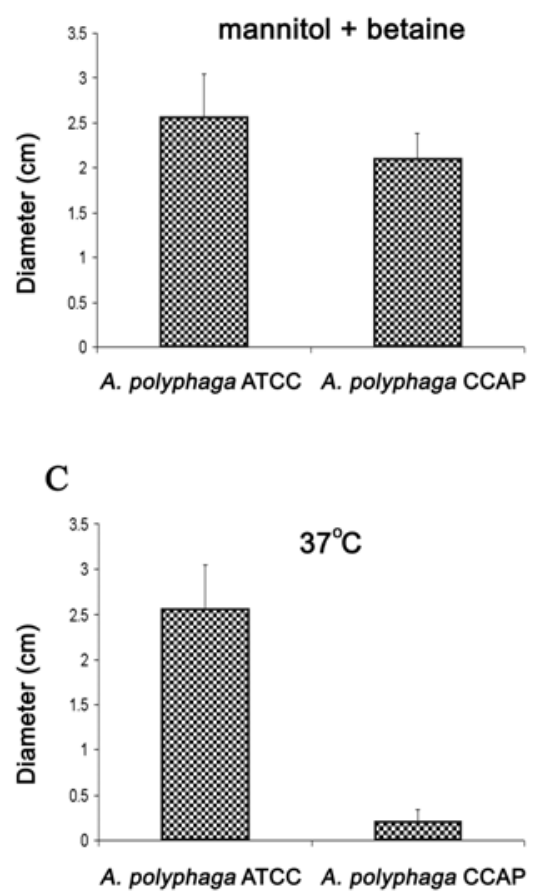

Fig. 3. Representative effects of high osmolarity and high temperature on the growth of Acanthamoeba polyphaga ATCC 30871 and A. polyphaga CCAP 1501/3c. A - Both Acanthamoeba isolates (approx. 1000 cells) were inoculated on nonnutrient agar plates overlaid with Klebsiella aerogenes, containing $1 \mathrm{M}$ mannitol at $30^{\circ} \mathrm{C}$ for up to $72 \mathrm{~h}$. Growth was observed microscopically as well as by measuring increase in cleared lawn of bacteria as described in "Materials and Methods". Note that plates inoculated with $A$. polyphaga ATCC 30871 exhibited clearance in bacterial lawn while $A$. polyphaga CCAP $1501 / 3 \mathrm{c}$ was not able to grow. B - Assays as described in (A) were performed in the presence of $1 \mathrm{mM}$ betaine (osmoprotectant). Note that both A. polyphaga ATCC and $A$. polyphaga CCAP $1501 / 3 \mathrm{c}$ were able to grow equally well. C - A polyphaga ATCC 30871 are temperature tolerant as compared to A. polyphaga CCAP 1501/3c. Both Acanthamoeba isolates were inoculated on non-nutrient agar plates overlaid with $K$. aerogenes and plates were incubated at $37^{\circ} \mathrm{C}$ for up to $72 \mathrm{~h}$. Results represent mean from three independent experiments. Each assay was performed in triplicates $(n=3)$, error bars represent standard deviation.
Acanthamoeba polyphaga ATCC 30871 but not CCAP $1501 / 3 \mathrm{c}$ is temperature- and osmotolerant

Previously we have shown that pathogenic Acanthamoeba strains are more temperature- and osmotolerant as compared to non-pathogens (Khan et al. 2001). To determine whether Acanthamoeba isolates used in this study can be differentiated based on osmolarity, assays were performed in the presence of $1 \mathrm{M}$ mannitol. Acanthamoeba polyphaga ATCC 30871 showed growth (thus clearance of bacterial lawns) in the presence of $1 \mathrm{M}$ mannitol while growth of $A$. polyphaga CCAP $1501 / 3 \mathrm{c}$ was inhibited (Fig. 3A). To test whether $A$. polyphaga CCAP $1501 / 3 \mathrm{c}$ growth inhibition was due to higher osmolarity, non-nutrient agar plates containing $1 \mathrm{M}$ mannitol were prepared in the presence of $1 \mathrm{mM}$ betaine (osmoprotectant). We observed that both amoeba isolates were able to grow on mannitol plates containing betaine thus confirming an osmotic effect (Fig. 3B). In temperature tolerance assays, we observed that $A$. polyphaga ATCC 30871 grew at $37^{\circ} \mathrm{C}$ while $A$. polyphaga CCAP 1501/3c did not (Fig. 3C).

\section{Isoenzyme analyses}

The isoenzyme patterns for esterase (ES), malate dehydrogenase (MDH), phosphoenol pyruvate dehydrogenase (PEPD) and isocitrate dehydrogenase (IDH) were distinct consistently between $A$. polyphaga ATCC 30871 and $A$. polyphaga CCAP $1501 / 3$ c (Fig. 4). Differences were observed in the mobilities of individual enzymes except in the case of $\mathrm{MDH}$, which showed differences both in the number and mobility of $\mathrm{MDH}$. Results are representative of at least three independent experiments. Only small differences were observed for the other isoenzymes tested (data not shown).

Acanthamoeba polyphaga ATCC 30871 and CCAP 1501/3c can be differentiated based on ARDRA

To determine the utility of ARDRA as a method for differentiating Acanthamoeba species, PCR-amplified rDNA was digested with the following enzymes: AluI, $C f o \mathrm{I}$, and Hinfl. Restriction patterns for each enzyme were distinct between $A$. polyphaga ATCC 30871 and A. polyphaga CCAP 1501/3c (Fig. 5), thus correlating well with other methods employed.

\section{DISCUSSION}

Methods for microbial species differentiation have evolved over the last few decades from simple culturing and microscopy to molecular methods such as rDNA sequencing. In this study we used morphological, biochemical, physiological and molecular methods to differentiate $A$. polyphaga ATCC 30871 and $A$. polyphaga CCAP $1501 / 3 \mathrm{c}$. We observed that although rDNA sequencing provided more characters for differentiation, all other methods employed were consistently effective in the differentiation of $A$. polyphaga ATCC 30871 and A. polyphaga CCAP 1501/3c.

Previously, morphological methods using microscopy have been used as a tool for the identification and 
PEPD

A. poly ATCC A. poly CCAP
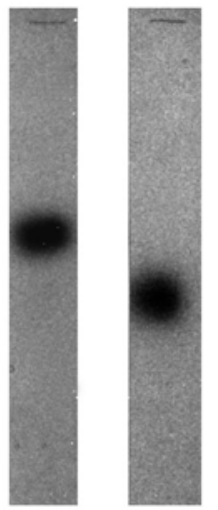

MDH

A. poly ATCC A. poly CCAP
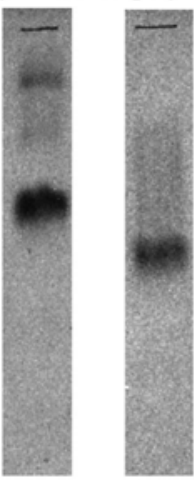

Esterase

A. poly ATCC A. poly CCAP
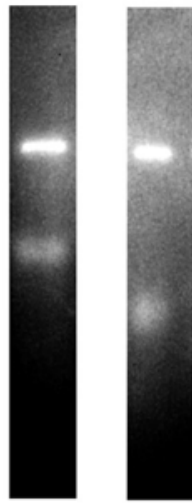

IDH

A. poly ATCC A. poly CCAP
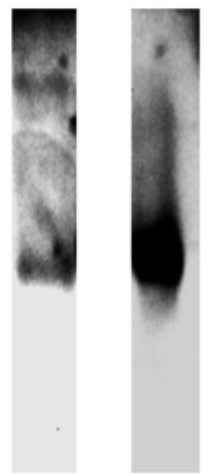

Fig. 4. Acanthamoeba polyphaga ATCC 30871 and A. polyphaga CCAP $1501 / 3 \mathrm{c}$ exhibited differences using isoenzyme analyses. Both Acanthamoeba isolates were tested for isoenzyme analyses as described in "Materials and Methods" and exhibited differences using phosphoenol pyruvate dehydrogenase (PEPD), esterase (ES), malate dehydrogenase (MDH) and isocitrate dehydrogenase (IDH). Results are representative of three independent experiments.

differentiation of Acanthamoeba based on cyst morphology (Pussard and Pons 1977, Visvesvara 1991). Acanthamoeba cysts are double-walled, consisting of an outer ectocyst and an inner endocyst. The endocyst is folded into arms or rays that protrude out from the cell body and contact the ectocyst at their ends. Based on cysts morphological analyses, the genus Acanthamoeba has been divided into three groups. Group 1 isolates of Acanthamoeba are characterised by large cysts with an average diameter of $>18 \mu \mathrm{m}$. In contrast, cyst isolates in groups 2 and 3 are $<18 \mu \mathrm{m}$. However, group 3 isolates possess three to five arms while number of arms in group 2 isolates is six or more. We determined that

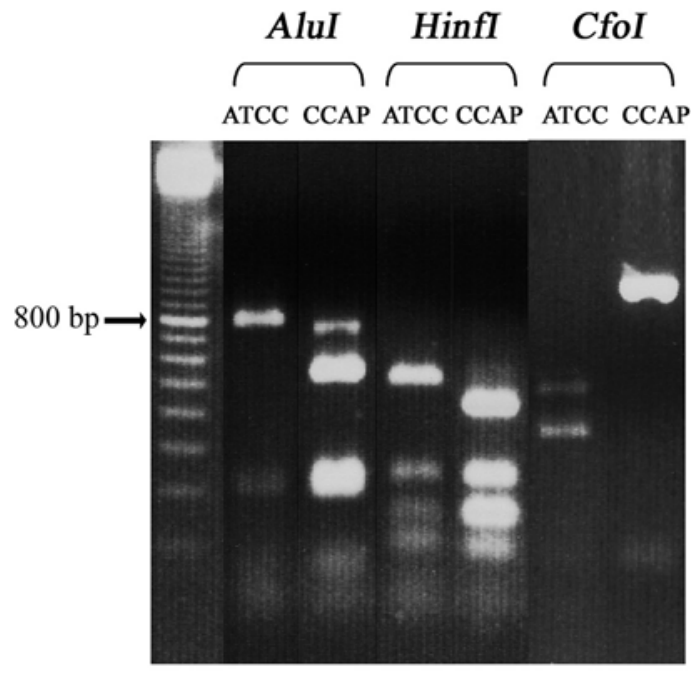

Fig. 5. Restriction analyses exhibited different banding patterns between Acanthamoeba polyphaga ATCC and A. polyphaga CCAP $1501 / 3 \mathrm{c}$. A PCR-amplified 18S rDNA Acanthamoeba-specific product was obtained from both $A$. polyphaga ATCC 30871 and CCAP 1501/3c. Restriction analyses were performed using AluI, CfoI, and Hinfl. Note that $A$. polyphaga ATCC 30871 and $A$. polyphaga CCAP 1501/3c can be differentiated with all restriction enzymes tested. Results are representative of three independent experiments.

cysts from both isolates of A. polyphaga ATCC 30871 and CCAP $1501 / 3 \mathrm{c}$ are $<18 \mu \mathrm{m}$ in size, suggesting that both isolates belong to either group 2 or 3 . To further determine grouping of these isolates, cysts were analysed for their number of arms. We observed that $A$. polyphaga ATCC 30871 exhibited up to six arms while A. polyphaga CCAP $1501 / 3 \mathrm{c}$ exhibited a maximum of five arms thus indicating their position in group 2 and 3 , respectively. Overall, we were able to differentiate both isolates of Acanthamoeba based on cyst morphology. However, microscopic identification is based solely on morphological characteristics, thus skill and use of robust keys for identification is required. In addition, correlating pathogenicity with particular species using morphology alone is not always reliable and this presents a problem for clinical diagnosis of pathogenic Acanthamoeba strains. Similar conclusions can be made from isoenzyme analyses. Using isoenzyme analyses, we were successfully able to differentiate $A$. polyphaga ATCC 30871 and CCAP $1501 / 3$ c using ES, MDH, PEPD and IDH. Previously, several groups have used analyses of isoenzyme electrophoretic patterns to test the morphological classification scheme. These studies discovered extensive diversity among isolates of Acanthamoeba. For example, De Jonckheere (1983), Daggett et al. (1985) and Costas and Griffiths (1986), studied large groups of isolates $(30,71$ and 37 strains, respectively). Each study divided isolates of Acanthamoeba into several different groups that often were 
inconsistent with species and morphological group designations. In addition, isoenzyme patterns have been shown to vary when amoebae are grown under different conditions i.e., axenic vs monoxenic (Weekers and De Jonckheere 1997). In addition, the requirement of a large number axenically grown parasites is also a shortcoming. In contrast, ARDRA is a sensitive method as amplifications can be achieved from a single cell (Abbaszadegan et al. 1991). This method is increasingly being used to identify parasitic organisms including Plasmodium (Waters and McCutchan 1989, Mathiopoulos et al. 1993), Leishmania (Uliana et al. 1991), Toxoplasma (Cazenave et al. 1991, Guay et al. 1993, MacPherson and Gajadhar 1993, Verhofstede et al. 1993), Entamoeba histolytica (Garfinkel et al. 1989, Bracha et al. 1990), Naegleria (Sparagano 1993), Cryptosporidium parvum (Laxer et al. 1991, 1992) and Giardia (Mahbubani et al. 1991, 1992). Usually ARDRA can be performed within 2-3 hours, and samples can be directly assayed without the prior need of cultivation making it a rapid assay. The restriction banding patterns depend on the nucleotide sequence recognised by restriction enzymes. Genetic polymorphism resulting even from a single base pair change can yield different electrophoretic patterns, if this change creates or eliminates a restriction enzyme site, offering a powerful and direct probe into the parasite genotype as observed in this study. Distinct restriction patterns were observed between $A$. polyphaga ATCC 30871 and CCAP $1501 / 3 \mathrm{c}$ using a number of restriction enzymes. Comparing restriction patterns from $A$. polyphaga CCAP $1501 / 3 \mathrm{c}$ with other strains of Acanthamoeba revealed that $A$. polyphaga CCAP $1501 / 3 \mathrm{c}$ gave similar patterns to A. palestinensis (data not shown). We further confirmed these differences in our study by rDNA sequencing of $A$. polyphaga CCAP $1501 / 3 c$, GenBank Ref. No. AF239298, which supported the conclusions from restriction enzyme analyses. Overall these methods have proven valuable and cost-effective in species differentiation of Acanthamoeba. However, these methods do not provide information to determine an isolate's pathogenic potential. Previously, we and others have shown that temperature tolerance (De Jonckheere 1980, Khan et al. 2001), osmotolerance (Khan et al. 2001), and extracellular proteases (Khan et al. 2000) can be used as potential pathogenic traits to determine an isolate's pathogenicity. Indeed, $A$. polyphaga ATCC 30871 trophozoites were able to grow at high temperatures, were osmotolerant and produced higher extracellular proteases (indicative of pathogenic strains), which was not observed with $A$. polyphaga CCAP 1501/3c, indicating that $A$. polyphaga CCAP $1501 / 3 \mathrm{c}$ may not possess pathogenic potential. To further confirm these findings, cytotoxicity assays were performed using corneal epithelial cells. Acanthamoeba polyphaga ATCC 30871 but not CCAP 1501/3c produced cytotoxicity on corneal epithelial cells in in vitro assays. At present, mechanisms of Acanthamoeba-induced cytotoxicity are incompletely understood but we have shown that pathogenic Acanthamoeba strains can be easily differentiated on the basis of these assays.

Over time we have observed the development of more detailed and reproducible methods for the differentiation of Acanthamoeba species. Here we demonstrate that morphological, biochemical, physiological and molecular methods have and will continue to serve as useful tools for species differentiation. Our data correlate well with the findings of Stothard et al. (1998), who based on rDNA sequencing showed that $A$. polyphaga ATCC and CCAP $1501 / 3 \mathrm{c}$ are distinct and reclassified the later as $A$. palestinensis (we further confirmed these differences in our study by rDNA sequencing, GenBank Ref. No. AF239297 [A. polyphaga ATCC 30871] and AF239298 [A. polyphaga CCAP 1501/3c]). In addition, $A$. polyphaga ATCC 30871 was placed among T4 pathogenic clade, while $A$. polyphaga CCAP $1501 / 3 \mathrm{c}$ was placed in T2 clade i.e., mostly weak and/or non-pathogens. When tested for their pathogenic traits (i.e., higher temperature tolerance, osmotolerance, extracellular proteases and in vitro cytotoxicity), we observed $A$. polyphaga ATCC 30871 to be pathogenic while $A$. polyphaga CCAP $1501 / 3$ c as non-pathogen thus correlating well with the data obtained by rDNA sequencing. In comparison with other assays, rDNA sequencing is more sensitive as more genetic characters are surveyed and thus serves as a sensitive marker of genetic change and is therefore more useful in identification of species and strains of the parasites. Our data together with these studies will aid in diagnostic and differentiation of pathogenic Acanthamoeba species. Ultimately, the identification and differentiation of pathogenic Acanthamoeba strains can be achieved by any one or combination of the methods described above promising to accelerate the pace of our understanding of this important and interesting class of pathogens.

Acknowledgements. This work was partially supported by grants from Faculty Research Fund, Birkbeck College, University of London. The authors greatly appreciate the support and guidance provided by Drs. Tim Paget (University of Hull) and Francesco Paolo Di Cello (Johns Hopkins University) throughout this project.

\section{REFERENCES}

ABBASZADEGAN M., GERBA C.P., ROSE J.B. 1991: Detection of Giardia cysts with a cDNA probe and applications to water samples. Appl. Environ. Microbiol. 57: 927-931.
AURAN J.D., STARR M.B., JAKOBIEC F.A. 1987: Acanthamoeba keratitis. A review of the literature. Cornea 6: 226. 
BADENOCH P.R., ADAMS M., COSTER D.J. 1994: Corneal virulence, cytopathic effect on human keratocytes and genetic characterization of Acanthamoeba. Int. J. Parasitol. 25: 229-239.

BRACHA R., DIAMOND L.S., ACKERS J.P., BURCHARD G.D., MIRELMAN D. 1990: Differentiation of clinical isolates of Entamoeba histolytica by using specific DNA probes. J. Clin. Microbiol. 28: 680-684.

CAZENAVE J., CHEYROU A., BLOUIN A., JOHNSON M., BEGUERET J. 1991: Use of polymerase chain reaction to detect Toxoplasma. J. Clin. Pathol. 44: 1037.

COSTAS M., GRIFFITHS A.J. 1986: Physiological characterization of Acanthamoeba isolates. J. Protozool. 33: 304-309.

DAGGETT P.M., LIPSCOMB D.S., THOMAS K., NERAD T.A. 1985: A molecular approach to the phylogeny of Acanthamoeba. Biosystems 18: 399-405.

DE JONCKHEERE J.F. 1980: Growth characteristics, cytopathic effect in cell culture, and virulence in mice of 36 type strains belonging to 19 different Acanthamoeba spp. Appl. Environ. Microbiol. 39: 681-685.

DE JONCKHEERE J.F. 1983: Isoenzyme and total protein analysis by agarose isoelectric focusing and taxonomy of the genus Acanthamoeba. J. Protozool. 30: 701-706.

DI GREGORIO C., RIVASI F., MONGIARDO N., DE RIENZO B., VISVESVARA G.S. 1991: Acanthamoeba meningoencephalitis in an AIDS patient: first report from Europe. Arch. Pathol. Lab. Med. 116: 1363-1365.

FRIEDLAND L.R., RAPHAEL S.A., DEUTSCH E.S., JOHAL J., MARTYN L.J., VISVESVARA G.S., LISCHNER H. 1992: Disseminated Acanthamoeba infection in a child with symptomatic human immunodeficiency virus infection. Pediatr. Infect. Dis. J. 11: 404407.

GARFINKEL L., GILADI M.I., HUBER M., GITLER C., MIRELMAN D., REVEL M., ROZENBLATT S. 1989: DNA probes specific for Entamoeba histolytica possessing pathogenic and nonpathogenic zymodemes. Infect. Immun. 57: 926-931.

GAST R.J. 2001: Development of an Acanthamoeba-specific reverse dot-blot and the discovery of a new ribotype. J. Eukaryot. Microbiol. 48: 609-615.

GONZALEZ M.M., GOULD E., DICKINSON G., MARTINEZ A.J., VISVESVARA G.S., CLEARY T.J., HENSLEY G.T. 1986: Acquired immunodeficiency syndrome associated with Acanthamoeba infection and other opportunistic organisms. Arch. Pathol. Lab. Med. 110: $749-751$.

GORDON S.M., STEINBERG J.P., DUPUIS M., KOZARSKY P., NICKERSON J.F., VISVESVARA G.S. 1992: Culture isolation of Acanthamoeba species and leptomyxid amebas from patients with amebic meningoencephalitis, including two patients with AIDS. Clin. Infect. Dis. 15: 1024-1030.

GUAY J.M., DUBOIS D., MORENCY J.M., GAGNON S., MERCIER J., LEVESQUE C.R. 1993: Detection of the pathogenic parasite Toxoplasma gondii by specific amplification of ribosomal sequences using comultiplex polymerase chain reaction. J. Clin. Microbiol. 31: 203207.
JOHN D.T. 1993: Opportunistically pathogenic free-living amebae. In: J.P. Kreier and J.R. Baker (Eds.), Parasitic Protozoa. Vol. 3. Academic Press, New York, pp. 143 246.

KHAN N.A. 2001: Pathogenicity, morphology and differentiation of Acanthamoeba. Curr. Microbiol. 43: 391-395.

KHAN N.A., JARROLL E.L., PAGET T.A. 2001: Acanthamoeba can be differentiated clinically by the polymerase chain reaction and simple plating assays. Curr. Microbiol. 43: 204-208.

KHAN N.A., JARROLL E.L., PANJWANI N., CAO Z., PAGET T.A. 2000: Proteases as markers of differentiation of pathogenic and non-pathogenic Acanthamoeba. J. Clin. Microbiol. 38: 2858-2861.

KONG H.H., CHUNG D.I. 1996: PCR and RFLP variation of conserved region of small subunit ribosomal DNA among Acanthamoeba isolates assigned to either A. castellanii or A. polyphaga. Korean J. Parasitol. 34: 127-134.

LAXER M.A., D’NICUOLA E.M., PATEL J.R. 1992: Detection of Cryptosporidium parvum DNA in fixed, paraffin-embedded tissue by the polymerase chain reaction. Am. J. Trop. Med. Hyg. 47: 450-455.

LAXER M.A., TIMBLIN K.B., PATEL J.R. 1991: DNA sequences for the specific detection of Cryptosporidium parvum by the polymerase chain reaction. Am. J. Trop. Med. Hyg. 45: 688-694.

MacPHERSON J.M., GAJADHAR A.A. 1993: Sensitive and specific polymerase chain reaction detection of Toxoplasma gondii for veterinary and medical diagnosis. Can. J. Vet. Res. 57: 45-48.

MAHBUBANI M.H., BEJ A.K., PERLIN M., SCHAEFER F.W., JAKUBOWSKI W., ATLAS R.M. 1991: Detection of Giardia cysts by using the polymerase chain reaction and distinguishing live from dead cysts. Appl. Environ. Microbiol. 57: 3456-3461.

MAHBUBANI M.H., BEJ A.K., PERLIN M., SCHAEFER F.W., JAKUBOWSKI W., ATLAS R.M. 1992: Differentiation of Giardia duodenalis from other Giardia spp. by using polymerase chain reaction and gene probes. J. Clin. Microbiol. 30: 74-78.

MARCIANO-CABRAL F., PUFFENBARGER R., CABRAL G.A. 2000: The increasing importance of Acanthamoeba infections. J. Eukaryot. Microbiol. 47: 29-36.

MATHIOPOULOS K., BOUARE M., McCONKEY G., McCUTCHAN T. 1993: PCR detection of Plasmodium species in blood and mosquitoes. In: D.H. Persing, T.F. Smith, F.C. Tenover and T.J. White (Eds.), Diagnostic Molecular Microbiology. American Society for Microbiology, Washington, D.C., USA, pp. 462-467.

NIEDERKORN J.Y., ALIZADEH H., LEHER H., McCULLEY J.P. 1999: The pathogenesis of Acanthamoeba keratitis. Microb. Infect. 1: 437-443.

PUSSARD M., PONS R. 1977: Morphologie de la paroi kystique et taxonomie du genre Acanthamoeba (Protozoa, Amoebida). Protistologica 13: 557-598.

SPARAGANO O. 1993: Differentiation of Naegleria fowleri and other Naegleriae by polymerase chain reaction and hybridization methods. FEMS Microbiol. Lett. 110: 325330. 
STOTHARD D.R., SCHROEDER-DIEDRICH J.M., AWWAD M.H., GAST R.J., LEDEE D.R., RODRIGUEZZARAGOZA S., DEAN C.L., FUERST P.A., BYERS T.J. 1998: The evolutionary history of the genus Acanthamoeba and the identification of eight new rRNA gene sequence types. J. Eukaryot. Microbiol. 45: 45-54.

ULIANA S.R.B., AFFONZO M.J.T., CAMARGO E.P., FLOETER-WINTER L.M. 1991: Leishmania: genus identification based on a specific sequence of the $18 \mathrm{~S}$ ribosomal RNA sequence. Exp. Parasitol. 72: 157-163.

VERHOFSTEDE C., RENIERS S., COLEBUNDERS R., WANZEELE F., PLUM J. 1993: Polymerase chain reaction in the diagnosis of Toxoplasma encephalitis, AIDS 7: 1539-1541.
VISVESVARA G.S. 1991: Classification of Acanthamoeba. Rev. Infect. Dis. 13 (S5): 369-372.

WATERS A.P., McCUTCHAN T.F. 1989: Rapid, sensitive diagnosis of malaria based on ribosomal RNA. Lancet i: 1343-1346.

WEEKERS H.H.P., DE JONCKHEERE J.F. 1997: Differences in isoenzyme patterns of axenically and monoxenically grown Acanthamoeba and Hartmannella. Antonie van Leeuwenhoek 71: 231-237.

YANG Z., CAO Z., PANJWANI N. 1997: Pathogenesis of Acanthamoeba keratitis: carbohydrate-mediated hostparasite interactions. Infect. Immun. 65: 439-445.

Accepted 19 November 2002 